\title{
REVIEW
}

\section{The role of epigenetics in the biology of multiple myeloma}

\author{
K Dimopoulos, P Gimsing and K Grønbæk
}

Several recent studies have highlighted the biological complexity of multiple myeloma (MM) that arises as a result of several disrupted cancer pathways. Apart from the central role of genetic abnormalities, epigenetic aberrations have also been shown to be important players in the development of MM, and a lot of research during the past decades has focused on the ways DNA methylation, histone modifications and noncoding RNAs contribute to the pathobiology of MM. This has led to, apart from better understanding of the disease biology, the development of epigenetic drugs, such as histone deacetylase inhibitors that are already used in clinical trials in MM with promising results. This review will present the role of epigenetic abnormalities in MM and how these can affect specific pathways, and focus on the potential of novel 'epidrugs' as future treatment modalities for MM.

Blood Cancer Journal (2014) 4, e207; doi:10.1038/bcj.2014.29; published online 2 May 2014

\section{INTRODUCTION}

Multiple myeloma (MM) is a clonal expansion of plasma cells, characterized by the production of a monoclonal protein, as well as end-organ damage. ${ }^{1}$ Virtually all cases of $M M$ are preceded by an indolent, premalignant disease known as monoclonal gammopathy of undetermined significance (MGUS) that can evolve to asymptomatic (or smoldering) MM and later to symptomatic $\mathrm{MM}^{2}$. The bone marrow microenvironment is thought to play a central role in the development of $M M$, but in the late stages of the disease, the malignant plasma cells become independent of this interaction by further acquiring new abnormalities that allow them to survive outside of the bone marrow, circulate in peripheral blood or migrate to other tissues, leading to plasma cell leukemia or extramedullary plasmacytomas, both considered more advanced stages of the disease. ${ }^{1}$

MM is a biologically complex disease, with great heterogeneity, reflected by its wide panel of genetic alterations and the individual differences in overall response and survival of patients receiving the same treatment. Genetic alterations, such as point mutations or translocations and deletions, as well as epigenetic alterations, such as aberrant DNA and histone methylation or abnormal microRNA (miRNA) expression, are found to contribute to the pathogenesis of $\mathrm{MM}^{3-5}$ Based on chromosomal alterations detected by karyotyping or fluorescent in situ hybridization, MM can be classified into two distinct groups: hyperdiploid, characterized by trisomies of odd-numbered chromosomes $(3,5,7,9,11,15,19$ and 21), and non-hyperdiploid, primarily characterized by translocations of $14 q 32$, but also gains of $1 p$ and monosomy 13.6 Some of these abnormalities also have an impact on prognosis, such as hyperdiploidy and $t(11 ; 14)$, both associated with a better prognosis, whereas $t(4 ; 14)$ or $\operatorname{del}(17 p)$ are associated with a worse prognosis. ${ }^{6}$

A wealth of research has been performed to elucidate the genetic aberrations seen in MM, but here we will focus on epigenetic abnormalities that are also central players in the disruption of common cancer pathways. It must be stressed that genetics and epigenetics are tightly connected and directly affect each other. It is now well established that DNA methylation and histone modifications can alter and control gene expression and, conversely, mutations affecting the function of epigenetic enzymes are described in numerous diseases, including MM. This review will focus on three common epigenetic mechanisms (aberrant DNA methylation, histone modifications and noncoding RNA expression) that cause abnormal signaling via critical pathways in MM, controlling cell cycle and apoptosis, and we will also briefly discuss the potential of epigenetic therapies in the context of MM.

\section{THE ROLE OF EPIGENETICS IN NORMAL CELLS AND IN THE DEVELOPMENT OF MM}

DNA methylation

The methylation of cytosine in the CpG (cytosine-phosphodiester bond-guanine) dinucleotide is the most studied epigenetic modification. Regions rich in CpG sites are known as CpG islands, and are often found in the promoter region and first exon of genes, as well as in repetitive elements, and it is now well known that DNA methylation of promoter $\mathrm{CpG}$ islands results in transcriptional inhibition and sometimes permanent gene silencing (Figure 1). ${ }^{7}$ The enzymes catalyzing the transformation of cytosine to 5-methylcytosine are known as DNA methyltransferases (DNMTs); DNMT3a and DNMT3b catalyze de novo DNA methylation, whereas DNMT1 catalyzes the maintenance of the DNA methylation after each cell division, assisted by DNMT3a and DNMT3b. ${ }^{8}$

The transcriptional silencing caused by DNA methylation was initially thought to be permanent, but recent studies have shown that demethylation of 5-methylcytosine is possible. The initial step is the addition of a hydroxyl group by the TET (ten-eleven translocation) proteins, and after additional covalent modifications and activation of base excision repair mechanisms, demethylation occurs. ${ }^{9}$ Thus, TET enzymes are suggested to ensure DNA methylation fidelity by keeping promoters of actively transcribed genes free of methylation. In addition, hydroxymethylation of cytosine might also play a role as a distinct epigenetic mark, but its precise function is yet to be elucidated. The regulation of gene expression by DNA methylation plays an essential role in cellular differentiation and tissue-specific gene expression, $\mathrm{X}$-chromosome 


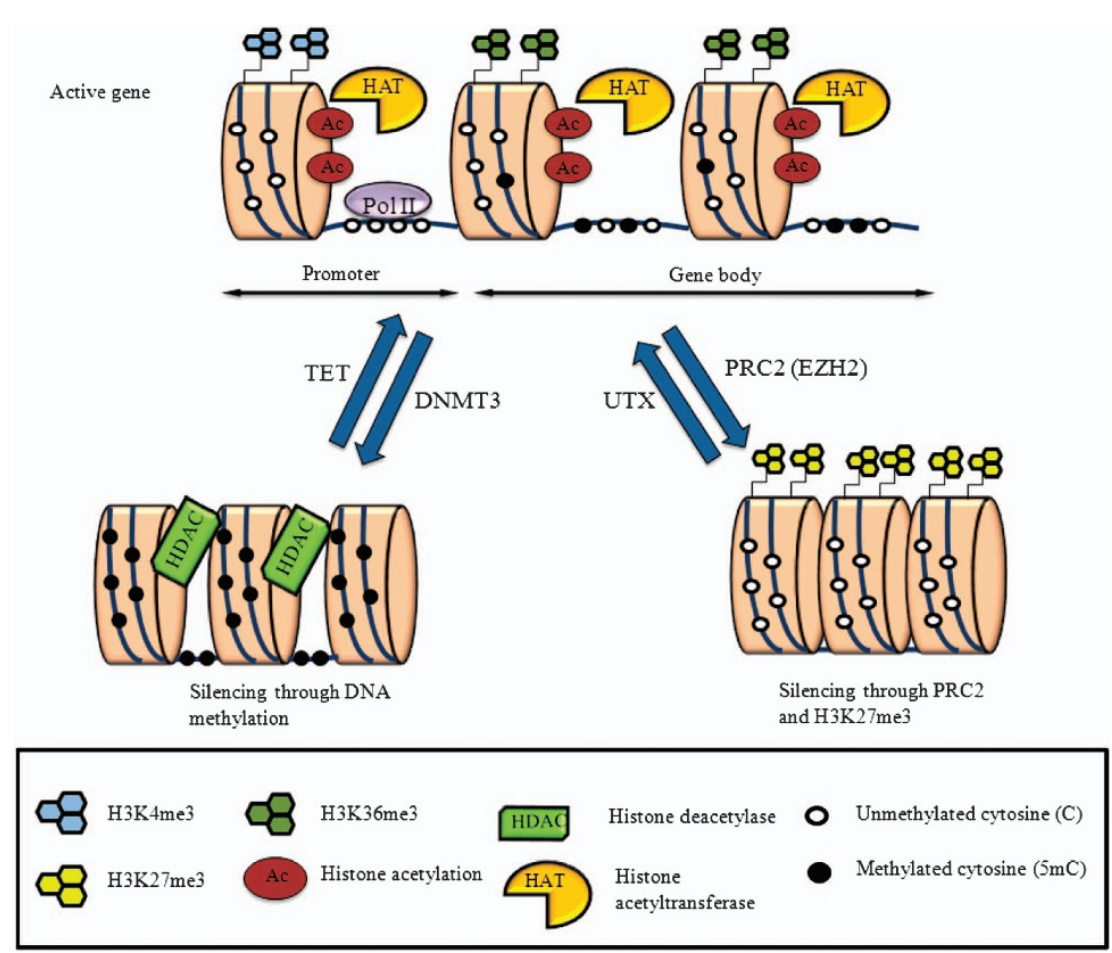

Figure 1. Transcriptionally active chromatin is characterized by histone acetylation, H3K4me3 and H3K79me3 in the promoter region (which is also nucleosome depleted), allowing binding of RNA polymerase II (Pol II), as well as H4K20me1 and H3K36me3 found in the body of transcriptionally active genes. At the same time, the CpG islands of the promoter region are unmethylated, and there is DNA methylation in the gene body. Gene silencing can occur with two different mechanisms: the first one involves methylation of the $\mathrm{CpG}$ islands of the promoter that then allows the binding of methyl-CpG-binding protein 2 (MeCP2) and recruitment of HDACs. Notably, DNA methylation does not affect histone methylation patterns. Gene silencing by DNA methylation was previously thought to be irreversible, but there is now evidence that TET proteins can actively demethylate 5 -methylcytosine $(5 \mathrm{mC})$ via the formation of $5 \mathrm{hmC}$. The histone methyl transferase $\mathrm{EZH} 2$ is the catalytic component of the PRC2 that causes H3K27me3-mediated gene silencing, independently of DNA methylation.

inactivation, genomic imprinting and silencing of transposable elements. In healthy cells, most CpG islands at transcription start sites are unmethylated, allowing gene expression, whereas methylation in gene bodies is often observed, and is related to increased transcriptional activity. ${ }^{7}$ Abnormal DNA methylation patterns are observed in most malignancies, where global DNA hypomethylation and gene-specific promoter hypermethylation is the main pattern. ${ }^{10}$

Several studies of DNA methylation in MM have revealed promoter hypermethylation of known tumor suppressor genes (Table 1). Genome-wide studies have confirmed global hypomethylation in MM with gene-specific hypermethylation, a common finding in most cancers. ${ }^{11-13}$ Although the data on the global DNA methylation patterns in MGUS seem to be conflicting, all studies seem to agree that throughout the course of the disease, the DNA methylation in promoter areas increases and reaches its height in plasma cell leukemia and human myeloma cell lines. ${ }^{11-13}$

The cause of aberrant DNA methylation patterns in $M M$ is currently unknown. The expression of DNMT1 is higher in MM compared with normal plasma cells and is increasing through the disease course. However, it is unknown whether the levels of DNMTs cause increased DNA methylation or is simply a marker of increased cell proliferation. MM has low expression of DNMT3a compared with normal plasma cells. This however is not explained by mutations or single nucleotide polymorphisms, but DNMT3A promoter hypermethylation has been reported in one study. ${ }^{12,14}$ In contrast to these findings, miR-29b, which is known to target DNMT3b, is frequently downregulated in $M M$, resulting in upregulation of DNMT3b, and an in vivo study in mice reported that transfection with synthetic miR-29b resulted in reduction of global DNA methylation and tumor growth inhibition. ${ }^{15}$

The function of TET proteins is regulated by a network that includes IDH1/2 (isocitrate dehydrogenase), which catalyzes the formation of $\alpha$-ketoglutarate, a co-factor for TET2, and miR-22, which directly targets TET2. ${ }^{16,17}$ Mutations in TET2 and IDH1/2 (isocitrate dehydrogenase) are frequent in glioblastoma and myeloid malignancies, ${ }^{18}$ but not reported in MM. However, Zhou et al. $^{19}$ found upregulation of miR-22 in MM by global miRNA expression, but this result is not confirmed in other studies.

\section{Histone modifications}

Histones are proteins found in all eukaryotic cells, and when packed with DNA they form units known as nucleosomes. The nucleosome consists of a histone octamer $(\mathrm{H} 2 \mathrm{~A}, \mathrm{H} 2 \mathrm{~B}, \mathrm{H} 3$ and $\mathrm{H} 4$, two pairs of each) around which $147 \mathrm{bp}$ of DNA are wrapped, resulting in a tight DNA packaging, and it is now known that the positioning of nucleosomes is yet another epigenetic modification that regulates gene expression. ${ }^{20}$ The nucleosomes together with DNA form the chromatin, and this may be further condensed to form chromosomes.

Post-translational modifications of histone tails, including methylation, acetylation, phosphorylation, ubiquitination, sumoylation and deimination among others, affect the interaction of DNA with histones and other DNA-binding protein complexes, thus playing important roles in the regulation of gene expression. ${ }^{21}$ The annotation of specific histone modifications includes the histone name, the amino acid and its specific position, and a short name of the modification; for example, 
Table 1. The different classes of histone deacetylases (HDACs) and their functions

\begin{tabular}{|c|c|c|}
\hline Classification & Localization & Comments \\
\hline \multicolumn{3}{|l|}{ Class I } \\
\hline HDAC1 & Nucleus & \multirow{4}{*}{$\begin{array}{l}\text { Ubiquitously expressed, } \\
\text { participate in } \\
\text { transcriptional regulation }\end{array}$} \\
\hline HDAC2 & Nucleus & \\
\hline HDAC3 & Nucleus & \\
\hline HDAC8 & Nucleus/cytoplasm & \\
\hline \multicolumn{3}{|l|}{ Class IIa } \\
\hline HDAC4 & Nucleus/cytoplasm & \multirow{4}{*}{ Tissue-specific functions } \\
\hline HDAC5 & Nucleus/cytoplasm & \\
\hline HDAC7 & Nucleus/cytoplasm & \\
\hline HDAC9 & Nucleus/cytoplasm & \\
\hline \multicolumn{3}{|l|}{ Class $I / b$} \\
\hline HDAC6 & Cytoplasm & \multirow[t]{2}{*}{$\begin{array}{l}\text { Formation of aggresome } \\
\text { and autophagy }\end{array}$} \\
\hline HDAC10 & Cytoplasm & \\
\hline \multicolumn{3}{|c|}{ Class III (sirtuins) } \\
\hline SIRT1 & Nucleus & \multirow{7}{*}{$\begin{array}{l}\text { Nicotine adenine } \\
\text { dinucleotide NAD +- } \\
\text { dependent lysine } \\
\text { deacetylases }\end{array}$} \\
\hline SIRT2 & Cytoplasm & \\
\hline SIRT3 & Mitochondria & \\
\hline SIRT4 & Mitochondria & \\
\hline SIRT5 & Mitochondria & \\
\hline SIRT6 & Nucleus & \\
\hline SIRT7 & Nucleus & \\
\hline \multicolumn{3}{|l|}{ Class IV } \\
\hline HDAC11 & Cytoplasm & $\begin{array}{l}\text { Role in interleukin (IL)-10 } \\
\text { expression }\end{array}$ \\
\hline
\end{tabular}

H3K27me3 refers to trimethylation of lysine on position 27 of histone 3.

Histone acetylation is a major regulatory mechanism of gene transcription and is balanced by the counteractive activity of two types of enzymes: histone acetyltransferases (HATs) and histone deacetylases (HDACs). HATs transfer an acetyl group from acetyl$\mathrm{CoA}$ to the lysine residues of histones, resulting in neutralization of their positive charge and interaction with the negatively charged phosphate groups of DNA, hence creating a less condensed chromatin form that allows binding of transcriptional factors; histone acetylation is thus associated with active gene expression. ${ }^{22}$ In contrast, HDACs remove acetyl groups from histone lysine residues back to acetyl-CoA, resulting in a condensed chromatin structure that is more condensed and transcriptionally inactive. ${ }^{22} \mathrm{~A}$ total of 11 HDACs have been discovered, and together with other proteins known as sirtuins, also showing HDAC activity, are divided in four subclasses (Table 1).

Histone methylation, on the other hand, is a more complex epigenetic mark than the 'on-off' acetylation changes. First, it exists as mono-, di- or tri-methylation of lysine residues, and as mono- and di-methylation of arginine residues on histones. Second, the level of methylation at a particular residue defines the function of the mark. For example, H3K4me3, H3K36me3, H3K79me3, H4R3me1 and H4K20me1 are associated with active gene transcription, whereas $\mathrm{H} 3 \mathrm{~K} 9 \mathrm{me} 3$ and $\mathrm{H} 3 \mathrm{~K} 27 \mathrm{me} 3$ are associated with gene silencing (Figure 1). ${ }^{23}$ The histone methyltransferase that catalyzes the methylation of H3K27me3, $\mathrm{EZH} 2$, is part of a protein complex known as polycomb repressive complex 2 (PRC2), and it is now acknowledged that PRC2 comprises a major mechanism of gene silencing. ${ }^{24}$ Even though earlier studies have also shown an interaction between $\mathrm{EZH} 2$ and DNMTs, possibly linking DNA methylation with $\mathrm{H} 3 \mathrm{~K} 27 \mathrm{me} 3$, these data are not confirmed and gene silencing through PRC2 and H3K27me3 is now considered a DNA methylation-independent mechanism of gene silencing (Figure 1). ${ }^{25}$ The distribution of histone methylation marks and the nucleosome positioning is also variable and dependent on the function and activity of the genomic area. For example, H3K4me3 and H3K79me3 are shown to be more prominent in active promoters, H3K4me1 is more often found in enhancer regions, whereas H4K20me1 and H3K36me3 locate to gene bodies of actively transcribed genes. ${ }^{23}$ However, recent data support the theory that individual histone changes are rarely adequate to influence gene expression, and it is their combinatorial patterns that regulate transcription. The first study to address this issue revealed 51 distinct chromatin states across human genome. ${ }^{26}$ Two other recent studies have reported over 800 different combinatorial patterns of histone changes, confirming the joint effect of histone modifications. ${ }^{27,28} \mathrm{~A}$ summary of the enzymes catalyzing the addition of epigenetic marks (writers), their removal (erasers) as well as of the enzymes that can bind and translate the epigenetic marks (readers) is given in Table 2.

Although the DNA methylation marks are relatively constant, the landscape of histone modifications is dynamic and constantly changing, and specific histone modifications influence each other, as for example ubiquitination of $\mathrm{H} 2 \mathrm{~B}$ is required for the methylation of H3K4me3. ${ }^{21}$ In addition, several studies have shown that DNA methylation and histone modifications often interact to regulate gene expression. DNA methylation allows the binding of methylCpG-binding protein 2 that then recruits HDACs that further deactivate gene transcription by condensing chromatin, and it is now clear that DNA methylation and histone acetylation interact closely to regulate gene expression ${ }^{29}$ (Figure 1).

Abnormal patterns of histone modifications are common findings in malignant cells. Mutations of histone-modifying enzymes and other epigenetic regulators have been reported in numerous cancers. ${ }^{30}$ Whole genome sequencing in MM has also revealed mutations in epigenetic regulators including UTX, MLL, MLL2, MLL3 and WHSC1, all resulting in an almost universally increased expression of $\mathrm{HOX}$, thus providing a novel therapeutic target. ${ }^{4}$

In MM, EZH2 upregulation can be mediated by interleukin- 6 (IL-6), by c-Myc activation, or be a consequence of miR-26a downregulation (which directly targets EZH2). Together with the aforementioned inactivating mutations of UTX, all these mechanisms can explain the increased levels of the H3K27me3 silencing signature at PRC2-targeted genes. ${ }^{31,32}$ Downregulation of PCAF (p300-CBP-associated factor), a histone acetyltransferase involved in the transcriptional control of several genes, including TP53, is caused by upregulation of the miR-106b-25 cluster in MM. ${ }^{33}$

However, the most characteristic and probably most interesting histone-modifying enzyme abnormality in the context of MM is the upregulation of MMSET (multiple myeloma SET domain) in all cases with $\mathrm{t}(4 ; 14)$, accounting for $\sim 15 \%$ of all patients. The MMSET gene (also known as NSD2 or WHSC1) is ubiquitously expressed in all tissues, and produces mainly two transcripts, known as MMSET-1 and MMSET-2. ${ }^{34}$ MMSET-2, which is the longer transcript, comprises four domains: PWWP (proline-tryptophan-tryptophanproline) domain, a HMG (high mobility group) box, a PHD (planthomeodomain) zinc finger and a SET domain. ${ }^{35}$ Early studies found MMSET to interact with both $\mathrm{H} 3$ and $\mathrm{H} 4$, and catalyzing H4K20 trimethylation, ${ }^{35,36}$ but now it is clarified that MMSET not only catalyzes the dimethylation of H3K36 (a H3K36 dimethylase), but also enhances the function of HDAC1 and HDAC2, and the histone demethylase LSD1 (Table 2). ${ }^{35,37-39}$ The result is global alterations of histone methylation patterns, with accumulation of H3K36me2 marks causing transcriptional activation of oncogenic loci, and a global reduction of H3K27me3 marks. ${ }^{37,38}$ This is in line with previous data showing that active histone marks, including H3K36me2, prevent binding of EZH2 to DNA. ${ }^{40}$

Increased expression of MMSET is also reported to contribute to the constitutive activation of nuclear factor- $\kappa \mathrm{B}$, a commonly 
Table 2. Epigenetic modifiers divided into categories according to their function ('writers', 'erasers' and 'readers') and the epigenetic mark they are related to

\begin{tabular}{|c|c|c|c|}
\hline Modification & Writers & Erasers & Readers \\
\hline Histone lysine acetylation & HATs & HDACs, SIRTs & Bromodomain proteins (BRD) \\
\hline \multirow{9}{*}{$\begin{array}{l}\text { Histone lysine methylation } \\
\text { H3K4 }\end{array}$} & KMT2A (MLL) & KDM1A (LSD1) & \multirow{24}{*}{ Chromodomain proteins (CHD) } \\
\hline & KMT2B (MLL4) & KDM1B (LSD2) & \\
\hline & KMT2C (MLL3) & KDM2B (CXXC2, JHDM1B) & \\
\hline & KMT2F (SETD1A) & KDM5C (JARID1C) & \\
\hline & KMT2G (SETD1B) & KDM5D (JARID1D) & \\
\hline & KMT3C (SMYD2) & NO66 (MAPJD) & \\
\hline & KMT3D (SMYD1) & & \\
\hline & KMT3E (SMYD3) & & \\
\hline & KMT7 (SETD7) & & \\
\hline \multirow[t]{5}{*}{ H3K9 } & KMT1A (Suv39H1) & KDM1A (LSD1) & \\
\hline & KMT8 (PRDM2) & KDM4D (JMJD2D) & \\
\hline & & KDM7 (JHDM1D) & \\
\hline & & PHF2 (JHDM1E) & \\
\hline & & PHF8 (JHDM1F) & \\
\hline \multirow{3}{*}{$\mathrm{H} 3 \mathrm{~K} 27$} & KMT6A (EZH2) & KDM6A (UTX) & \\
\hline & KMT6B (EZH1) & KDM6B (JMJD3) & \\
\hline & & KDM7 (JHDM1D) & \\
\hline \multirow[t]{7}{*}{ H3К36 } & KMT2H (ASHL1) & KDM2A (CXXC8, JHDM1A) & \\
\hline & KMT3A (SETD2) & KDM2B (CXXC2, JHDM1B) & \\
\hline & КMT3B (NSD1) & KDM4A (JMJD2A, JHDM3A) & \\
\hline & KMT3C (SMYD2) & KDM4B (JMJD2B) & \\
\hline & NSD2 (MMSET) & KDM4C (JMJD2C, JHDM3C) & \\
\hline & SETMAR (Metnase) & KDM4D (JMJD2D) & \\
\hline & & KDM8 (JMJD5) & \\
\hline
\end{tabular}

Abbreviations: DNMT, DNA methyltransferase; HAT, histone acetyltransferase; KDM, lysine demethylase; KMT, lysine methyltransferase; MBD, methyl-CpGbinding domain; MeCP2, methyl-CpG-binding protein 2.

disrupted pathway in $\mathrm{MM}^{41}$ and inhibition of MMSET in vitro reduces cell growth of the malignant cells, providing a potential

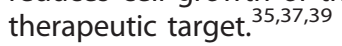

\section{Noncoding RNAs}

It has been shown that although only $1-2 \%$ of the human genome consists of protein-encoding DNA, up to $75 \%$ of our DNA is transcriptionally active. ${ }^{42}$ This DNA, which was previously considered 'junk-DNA', produces noncoding RNA (ncRNA) molecules, some of which play central regulatory roles in gene expression, and control important cellular functions. The ncRNAs are divided into two major groups, according to the size of the transcript: long ncRNAs (>200 nt) and small ncRNAs (18-200 nt). The latter category comprises miRNAs, small interfering RNAs, small nucleolar RNAs, small nuclear RNAs, PIWI-interacting RNAs and vault RNAs. The most extensively studied group of ncRNAs is miRNAs, small transcripts (18-22 nt) originating from either intragenic or intergenic DNA loci, and they are known to posttranscriptionally regulate gene expression. ${ }^{43}$ The biogenesis of miRNA in mammals is complex and reviewed in detail elsewhere. ${ }^{5}$
The importance of miRNAs in the regulation of functions such as cell differentiation, development and apoptosis was highlighted early after their discovery, and it is now known that aberrant miRNA expression is seen in many human diseases, including cancer, where miRNAs can act as oncogenic miRNAs or as tumor suppressors. Even though miRNAs are not considered a true epigenetic regulatory mechanism, they interact closely with other critical epigenetic regulators, for example the miR-29 family with DNMT3A and DNMT3B, miR-26a with EZH2 and miR-449a with HDAC1. ${ }^{32,44,45}$ On the other hand, epigenetic regulation of miRNAs is extremely common, and epigenetic silencing of miRNAs in MM through promoter hypermethylation has been reported. ${ }^{46-48}$ The role of miRNAs in the development of MM has been highlighted in several studies. ${ }^{19,33,49,50}$ Commonly deregulated miRNAs in MM include miR-21, the miR-17-92 cluster, the miR-15a/16 cluster and the miR-29 and miR-34 families, all of which are also found deregulated in other malignancies. Data indicate that MGUS and MM share a common miRNA signature that distinguishes them from normal plasma cells, but MM cells exhibit additional deregulated miRNAs compared with MGUS. ${ }^{33}$ In addition, some miRNAs are associated with drug resistance in 
myeloma, possibly through downregulation of key antimyeloma drug targets; alternatively, downregulation of miRNAs throughout disease progression may lead to upregulation of critical myelomarelated genes. ${ }^{51}$

The role of other types of ncRNAs in MM has barely been studied. Ronchetti et $a l^{52}$ recently reported deregulation of small nucleolar RNAs in MM, and were able to correlate differential small nucleolar RNA expression within previously defined gene expression profiling subgroups. However, little is known about the role of long ncRNAs in myeloma, a subject that could be of interest.

\section{EPIGENETICS ALTER CRITICAL REGULATORY NETWORKS IN MM}

Each of the aforementioned alterations of the epigenetic machinery may result in dysregulation of critical oncogenic pathways that contribute to the pathogenesis of MM. A description of the most commonly involved networks and their disruption through abnormal DNA methylation, histone modifications or altered miRNA expression is as follows.

\section{Cyclin/CDK/Rb pathway}

Tight control of cell cycle is essential for normal cellular growth, and disrupted cell cycle regulation may promote carcinogenesis. In the G1 phase, CDK4 and CDK6 (cyclin-dependent kinases 4 and 6) get activated and form complexes with Cyclin D molecules (D1, D2 and D3), causing phosphorylation and deactivation of the $\mathrm{Rb}$ (retinoblastoma) protein, an event that activates the CDK2/Cyclin A/E complex and members of the E2F family, finally resulting in the transition from $\mathrm{G} 1$ to $\mathrm{S}$ phase (Figure 2). Negative regulators of this process are called CKIs (cyclin-dependent kinase inhibitors) and include members of the INK4 family ( 16 INK4a encoded by CDKN2A, p15INK4b encoded by CDKN2B, p18INK4c encoded by CDKN2C and p19INK4d encoded by $C D K N 2 D)$ that inhibit CDK4/6, and members of the Cip/Kip family (p21Cip1 encoded by CDKN1A, p27Kip1 encoded by $C D K N 1 B$, and p57Kip2 encoded by CDKN1C) that inhibit CDK2.
The CDKN2A gene has two different coding frames: the INK4A that encodes p16INK4a by splicing of exons $1 \alpha, 2$ and 3 , and $A R F$ (alternative reading frame) with its own promoter, encoding p14 ${ }^{\mathrm{ARF}}$ (after splicing of exons $1 \beta$ and 2). In contrast to p16INK4a, $\mathrm{p} 14^{\mathrm{ARF}}$ is not directly involved in the control of cyclin D/CDK4/6, but interacts with $M D M 2$, resulting in stabilization of $p 53$ and cell cycle arrest via p21/CDK2/cyclin E inhibition. ${ }^{53}$

Disruption of this fine regulatory system has been associated with cancer development, and several tumors exhibit overexpression of cyclin D members and CDK4, or loss of Rb. ${ }^{54}$ Like $\mathrm{Rb}$, all CKIs are considered to be tumor suppressors, and their functional loss can contribute to tumorigenesis. Among all CKIs, p16INK4a is the most widely studied in cancer, and point mutations, deletions and epigenetic silencing are seen in a variety of human cancer cell lines and primary tumors. ${ }^{55}$

In myeloma, overexpression of cyclin D members occurs in most cases, and is considered to be a common and early pathogenic event in the development of the disease. Translocations including the IGH locus on $14 q 32$ can result in direct upregulation of cyclin genes when involving $11 q 13$ (cyclin D1) or 6 p21 (cyclin D3). ${ }^{56}$ No genetic or epigenetic alterations are reported for CDK4 or other CDKs in MM, but miR-29b and the miR-34 family, which under normal circumstances regulate the levels of CDK6, cyclin D1 and CDK4, are reported to be downregulated in $M M$, resulting in upregulation of the cyclin D/CDK4/6 complex (Figure 3). ${ }^{48,57}$ Interestingly, the downregulation of the $\mathrm{miR}-34 \mathrm{~b} / \mathrm{c}$ in many cases of MM, especially later in the course of the disease, is found to be a result of promoter hypermethylation of the MIR34B/C gene. ${ }^{47}$ Furthermore, it is shown that the miR-17-92 cluster, which is upregulated in $\mathrm{MM}$, targets E2F1, allowing increased cell proliferation. $^{33,58}$ Whereas $\mathrm{Rb}$ deletions associated with del(13q) are detected in up to half of the cases, downregulation of $R B 1$ by promoter hypermethylation is not seen in $\mathrm{MM}^{59}$

Several studies have addressed the issue of downregulation of CKIs by promoter hypermethylation in MM. Inactivating mutations or deletions of CDKN2A are rare in MM, but promoter hypermethylation of the INK4A promoter occurs in $\sim 40 \%$ of the patient

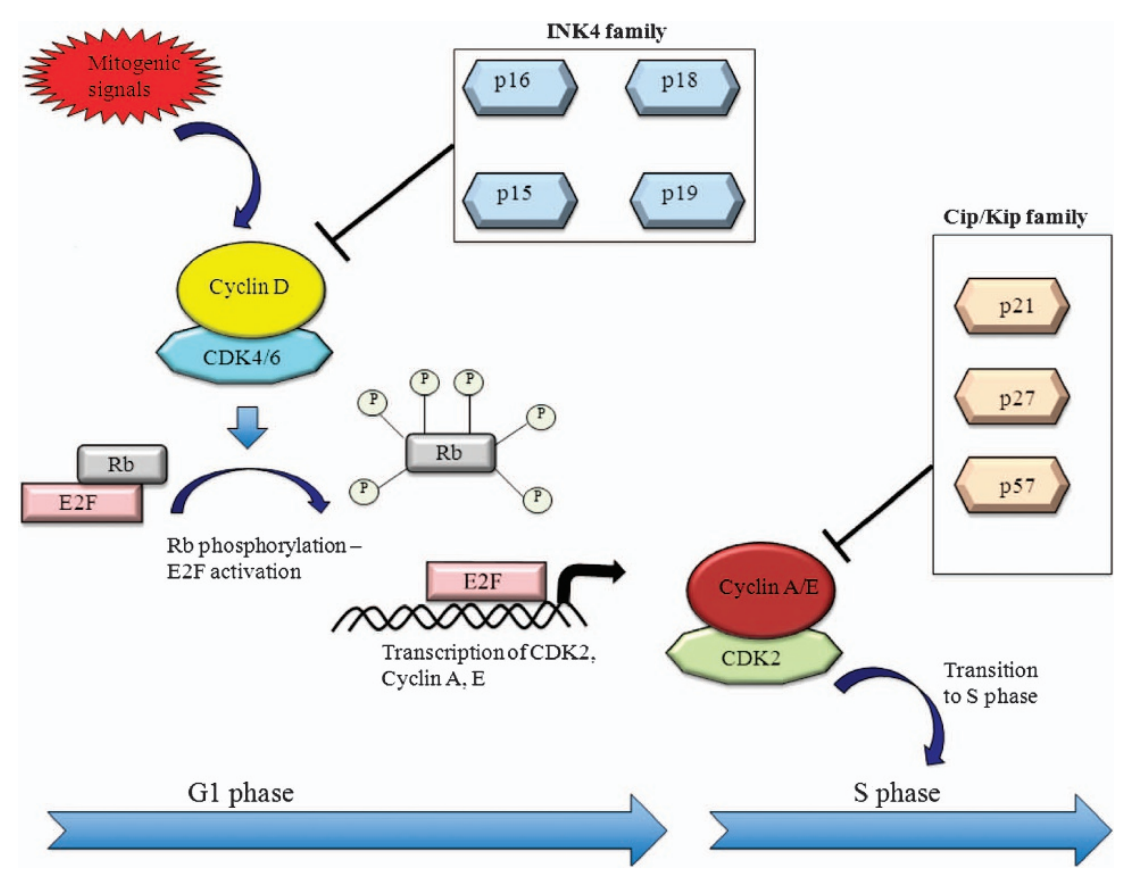

Figure 2. The cyclin/CDK pathway. The entry in G1 phase is characterized by the assembly of CDK4/6 and cyclin D proteins, a process controlled by the INK4 family of CKIs. The cyclin D/CDK4/6 complex phosphorylates and deactivates Rb, allowing E2F to activate transcription of CDK2 and cyclins $A$ and $E$ that are needed for the transition to the $S$ phase. The cyclin A/E/CDK2 complex is inhibited by the Cip/Kip family of CKIs. 


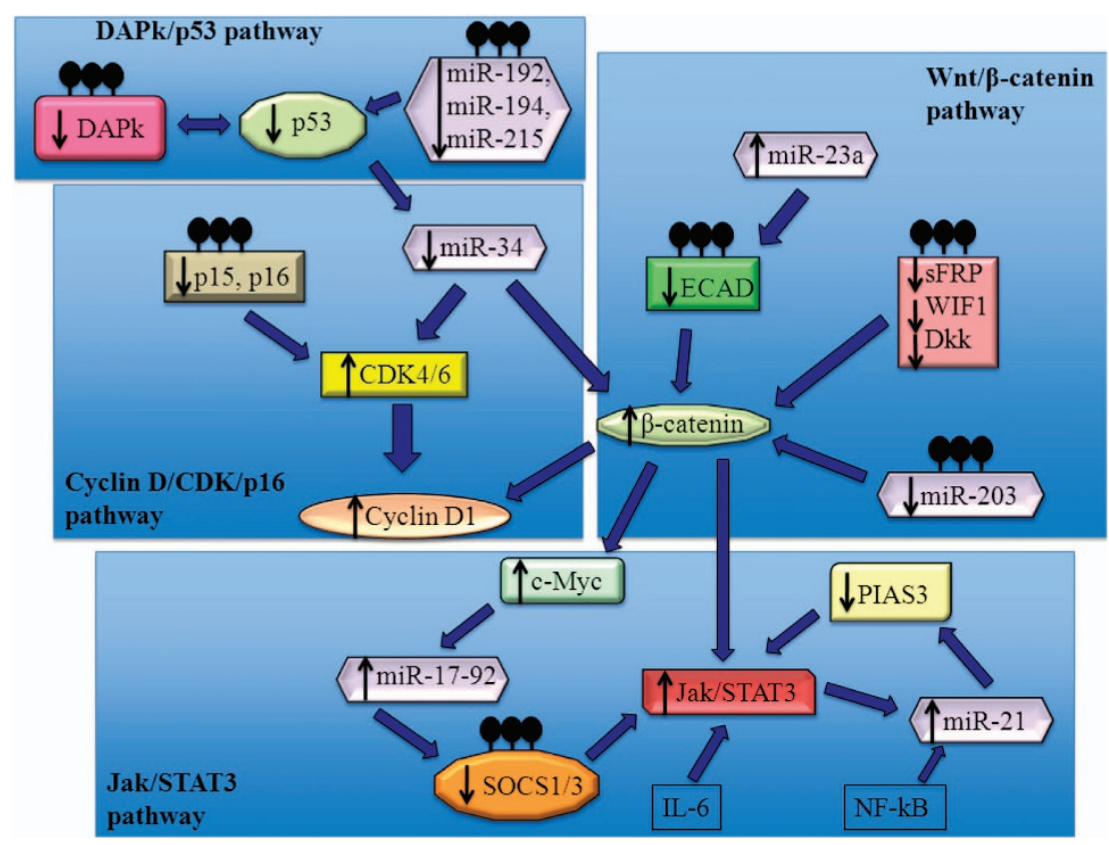

Figure 3. Combination of promoter hypermethylation of tumor suppressor genes acting as inhibitors of cancer pathways as well as abnormal expression of miRNAs contribute to the activation of the cyclin D/CDK pathway, Jak/STAT3 pathway, Wnt/ $\beta$-catenin signaling pathway and disruption of DAPk/p53 interaction. The dark circles above genes and miRNAs indicate DNA methylation and silencing of the respective gene/miRNA.

Table 3. Tumor suppressor genes whose promoter is found to be hypermethylated in MGUS/MM in two or more studies

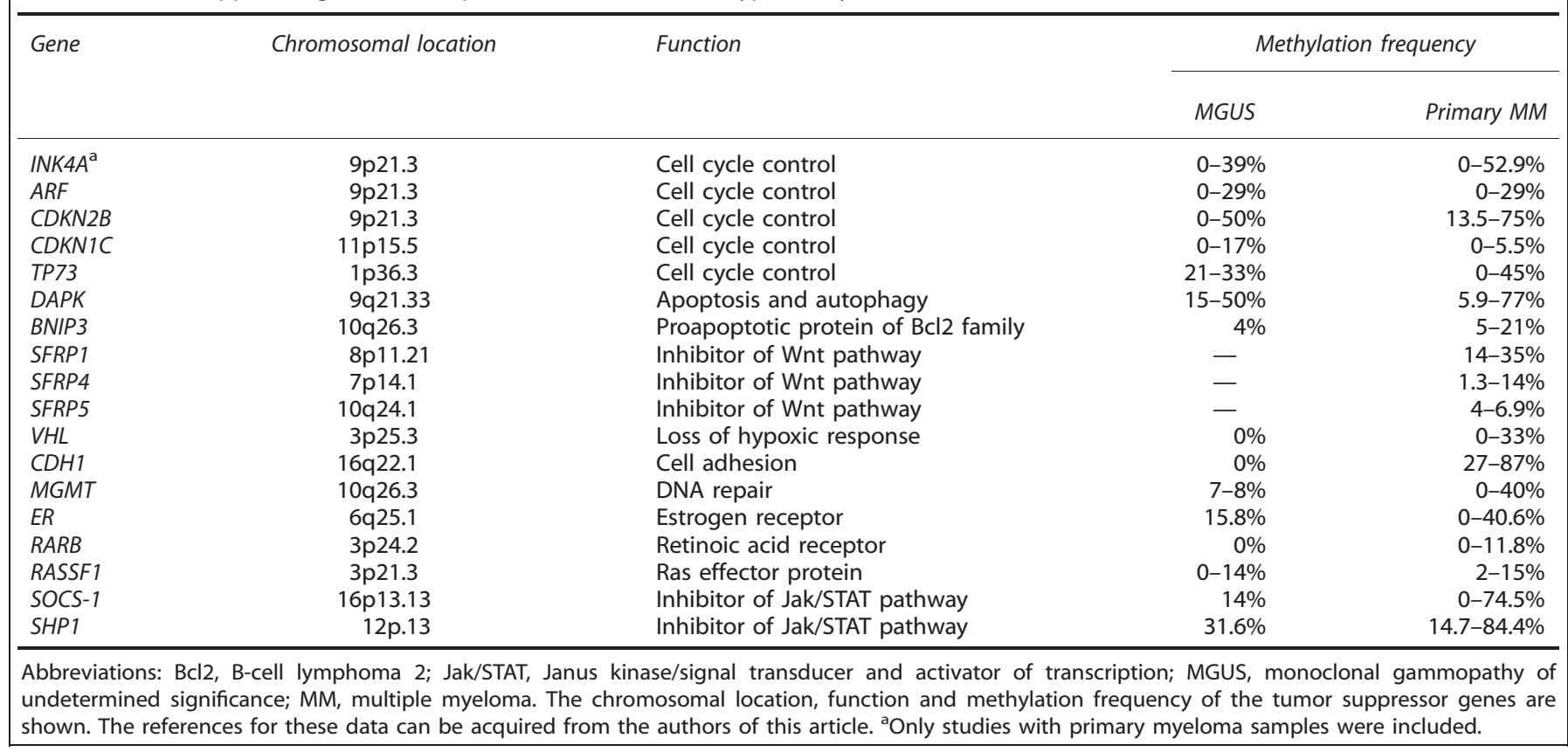

samples with newly diagnosed MM, although the reported methylation frequency is extremely variable (Table 3) probably because of different methodologies. Similarly, promoter hypermethylation of CDKN2B is reported in MM, ranging from just above $10 \%$ to over $80 \%$ of the MM patient samples examined, whereas promoter methylation of CDKN2C or members of the Cip/Kip family is infrequent in MM (Table 3).

It is unclear whether INK4A promoter methylation is associated with biological or clinical features of $\mathrm{MM}$, as its association with overall survival disease stage has yielded conflicting data. ${ }^{60-63}$ Moreover, it seems that promoter methylation of INK4A is not the only contributor to p16 downregulation, as low expression is seen in almost all cases of MM, even those without promoter methylation. ${ }^{61,64}$ This suggests that either the promoter methylation of the CDKN2A does not have any biological significance in $\mathrm{MM}$, being a 'secondary' epigenetic event, or that there are mechanisms that downregulate p16 other than promoter hypermethylation, such as ncRNAs, histone modifications or lack of the appropriate transcription factors.

\section{THE WNT/ $\beta$-CATENIN PATHWAY AND E-CADHERIN}

Wnt signaling controls multiple functions including cell migration during embryonic development, hematopoiesis and tissue 
homeostasis. ${ }^{65}$ It consists of the $\beta$-catenin-dependent pathway, involved in cell cycle control, and the $\beta$-catenin-independent pathway, involved in planar cell polarity pathway and intracellular calcium regulation (Wnt-calcium pathway). ${ }^{65}$ Disruption of the Wnt pathway, especially the $\beta$-catenin-dependent pathway, is associated with a series of developmental diseases, and also cancer. $^{66}$

Activation of the Wnt pathway results in intranuclear accumulation of $\beta$-catenin, allowing its binding with T-cell factor/lymphoid enhancer factor 1 (LEF1) and formation of a transcription factor complex that activates the transcription of several genes. Some of the known target genes that are transcriptionally activated by $\beta$-catenin/transforming growth factor (TGF)/LEF1 include CCDN1 (coding for cyclin D1), STAT3 and MYC, all known to be upregulated in MM. ${ }^{66}$ Finally, the $\beta$-catenin/TGF/LEF complex is found to downregulate the expression of p16INK4a (suggesting another mechanism for p16 downregulation apart from methylation), as well as of miR-15a/16, resulting in both further upregulation of cyclin D1 and increased angiogenesis in MM. ${ }^{67-69}$

The Wnt pathway is inhibited by several proteins, divided into two subclasses: the sFRP (secreted Frizzled-related protein) class, including the sFRP family, WIF-1 (Wnt inhibitory factor 1) and Cerberus, all of which bind directly to Wnt ligands, and the Dickkopf class, which includes the Dickkopf (Dkk) protein family that inhibit the Wnt pathway by binding to LRP5/6. ${ }^{70}$ DKK 1 is of special interest in MM, as it has been shown to be associated with myeloma bone disease. ${ }^{71}$ Promoter hypermethylation of SFRP1, SFRP2, SFRP3, SFRP5, DKK3, APC and WIF1 and subsequent activation of Wnt pathway in MM patients has been described in MM (Table 3). Moreover, it is shown that miR-34a, which is often downregulated in MM (though not by promoter hypermethylation), is a direct inhibitor of the Wnt pathway. ${ }^{72}$ MiR-203 is another miRNA that inhibits the Wnt pathway and whose promoter has been found to be hypermethylated in MM (Figure 3). ${ }^{46,73}$ Interestingly, even though miR-21 and miR-200a are also known to act as inhibitors of the Wnt signaling pathway, both are found upregulated in MM. ${ }^{49,50,67}$ However, it is well-known that the function of miRNAs is highly cell type dependent. HDACs are also reported to interfere with $\beta$-catenin signaling; HDAC1, HDAC2 and HDAC7 inhibit whereas HDAC3 and HDAC6 activate the Wnt pathway. ${ }^{73}$

Apart from the cytoplasmic pool of $\beta$-catenin that regulates gene transcription through the Wnt pathway, $\beta$-catenin is also bound to the cytoplasmic domain of E-cadherin (ECAD), a protein responsible for intercellular adhesion. Loss of $\mathrm{CDH} 1$ (the gene coding for ECAD) is associated with increased tumor invasion and contributes to metastases. ${ }^{74}$ Furthermore, there is a dynamic interaction between cadherin/catenin complex and Wnt/ $\beta$-catenin, and recent studies confirm that cadherin-bound $\beta$-catenin can be released into the cytoplasm and act as a transcriptional activator. ${ }^{75}$

Promoter hypermethylation of the $\mathrm{CDH} 1$ is not detected in patient samples with MGUS, whereas the methylation frequency in $\mathrm{MM}$ is reported to be $27-92 \%$ in primary samples, suggesting that methylation of $\mathrm{CDH} 1$ might contribute to the development of MM from MGUS (Table 3). In some of these studies, higher frequency of $\mathrm{CDH} 1$ promoter hypermethylation is reported in relapsed MM and plasma cell leukemia, and it is furthermore shown to be an acquired event through the disease course. ${ }^{63,76}$ It is unknown whether the epigenetic inactivation of ECAD also results in constitutive Wnt signaling in MM. Targeting of ECAD by miRNAs has also been reported; ECAD is downregulated by miR-23a in lung cancer cells, a miRNA also found upregulated in MM (Figure 3). ${ }^{19,33,77}$

\section{IL-6 AND JAK/STAT SIGNALING}

It is now well defined that increased secretion of IL-6 with consequent activation of the IL- 6 receptor and Janus kinase/signal transducer and activator of transcription (JAK/STAT3) pathway is essential for the antiapoptotic properties of malignant plasma cells in the development of MM. Known inhibitors of the JAK/STAT3 pathway include among others the protein tyrosine phosphatases, the SOCS (suppression of cytokine signaling) and PIAS (protein inhibitor of activated STAT) protein families. ${ }^{78}$

Promoter hypermethylation of genes coding for inhibitors of the JAK/STAT pathway, including SHP1, SOCS1 and SOCS3, is reported in varying frequencies in MM (Table 3). Another mechanism of increased JAK/STAT signaling in $M M$ is the upregulation of the miR-17-92 cluster that directly targets SOCS- 1 and SOCS-3. ${ }^{33,79}$ There is, to date, no reported promoter hypermethylation of the genes coding for the PIAS protein family; however, PIAS3 is a direct target of miR-21, a miRNA that is directly upregulated in MM by both nuclear factor- $\kappa B$ and STAT3, leading to further JAK/STAT3 activation in plasma cells and resistance to apoptosis (Figure 3 ). ${ }^{80-82}$

Finally, HATs and HDACs are also shown to regulate JAK/STAT3 signaling, as STAT3 is reported to be activated by acetylation catalyzed by the HAT CBP/p300, and activated STAT3 regulates gene expression through recruitment of HDAC1 that in turn inhibits STAT3 in a negative regulatory loop. ${ }^{83}$

\section{DAPk/P14 ${ }^{\text {ARF } / p 53 ~ P A T H W A Y ~}$}

The death-associated protein kinase (DAPk) family includes three members: DAPk (or DAPk1), DRP-1 (or DAPk2) and ZIPk (or DAPk3), all of which control apoptosis and autophagy. The most extensively studied protein of this family is DAPk, as it is often downregulated in cancer, allowing malignant cells to escape apoptosis. The antiapoptotic function of DAPk is closely related to p53. Oncogenic stimuli such as c-Myc or E2F2 have been shown to initially activate p53, leading to transcriptional activation of DAPk that in turn stabilizes p53 in a positive feedback loop through upregulation of $\mathrm{p} 14^{\mathrm{ARF}}$, finally resulting in cell cycle arrest and apoptosis. $^{84}$

As mentioned above, promoter hypermethylation of $A R F$ is not reported in either MGUS or MM, but DAPK promoter hypermethylation is commonly seen in MM (Table 3). TP53 mutations are rare in $\mathrm{MM}$, and are only seen in patients with del(17p), whereas promoter hypermethylation is also infrequent and has not been reported in patient samples. However, several miRNAs seem to regulate the $\mathrm{p} 53$ axis in $\mathrm{MM}$, including the miR-106b-25 cluster and miR-192, miR-194 and miR-215. ${ }^{33,48}$ The last three miRNAs target MDM2, an inhibitor of p53, and they are downregulated in MM by promoter hypermethylation. ${ }^{48}$

\section{EPI-THERAPEUTICS: A NOVEL TREATMENT MODALITY IN MM}

The past decades have seen a massive development of new cancer drugs, some of them acting by changing the epigenome in the malignant cells. The DNMT inhibitors 5-azacytidine and 5-aza2 'deoxycytidine are already approved for the treatment of higherrisk myelodysplastic syndromes and acute myeloid leukemia with low blast counts. ${ }^{85}$ Although in vitro studies have confirmed antimyeloma activity of 5 -azacytidine, ${ }^{86}$ the in vivo effects of DNMT inhibitors in MM are yet to be examined in clinical trials.

Another class of epigenetic drugs thoroughly examined in the treatment of myeloma are the HDAC inhibitors (HDACis). The specificity of HATs and HDACs for histones is not high, and they are known to modify the acetylation status of other proteins, such as nuclear factor- $\mathrm{KB}, \mathrm{p} 53$ and STAT proteins, thus affecting their stability and functions. ${ }^{87}$ Accordingly, it is clear that HDACis not only have an effect on transcriptional regulation through histone modifications, but also influence the activity of several transcription factors in pathways that are disrupted in $M M$, as shown in numerous functional studies. ${ }^{88,89}$ However, clinical trials have failed to show a clinical benefit with HDACis as single-agent 
therapy, with the exception of Panobinostat; nevertheless, a synergistic effect of HDACis with bortezomib and immunomodulatory drugs has been reported. ${ }^{90-92}$ The inefficacy of single-agent HDACi treatment is not only seen in myeloma, but also in several malignancies, and could be because of the inability of HDAC inhibition alone to induce apoptosis in vivo. More likely, HDACis allow the transcription of genes that are necessary for other drugs to function. Thus, the synergy between proteasome inhibitors or immunomodulatory drugs could be a result of increased DNA damage and apoptosis in the malignant plasma cells. Alternatively, HDACis may further enhance the inhibition of HDAC expression caused by bortezomib.

An interesting therapeutic target is HDAC6, a class Ilb cytoplasmic HDAC that plays a central role in the degradation of misfolded proteins through autophagy, an alternative mechanism to proteasome degradation. ${ }^{93}$ Simultaneous inhibition of the proteasome function and HDAC6 can thus lead to accumulation of misfolded proteins and apoptosis, and could possibly explain the synergy between bortezomib and HDACis. A selective HDAC6 inhibitor, ACY-1215, is a promising epigenetic targeted therapy for myeloma that has shown in vitro and in vivo effectiveness ${ }^{94}$ and is now tested in two ongoing phase 1-2 clinical trials (ClinicalTrials.gov. NCT01323751 and NCT01583283).

Histone methylation-modifying drugs (lysine demethylase inhibitors and/or lysine methyltransferase inhibitors) are under development, and their efficacy has not yet been studied in in vivo models. ${ }^{95}$ An interesting target is MMSET in the myeloma subtype with $\mathrm{t}(4 ; 14)$, as it is upregulated in all these patients. MMSET exhibits its activity through its PHD domain, as artificial mutations in the PHD domain inactivated the methyltransferase activity of MMSET. ${ }^{39}$ Thus, targeting of the PHD domain of MMSET may be attractive in the development of new epigenetic drugs. However, no known MMSET inhibitors are available, but their development could potentially alter the standard treatment and prognosis of myeloma cases with $\mathrm{t}(4 ; 14)$.

Finally, novel drugs targeting the 'readers' of epigenetic marks are under development. A promising example is JQ1, an inhibitor of the bromodomain protein BRD4, that in a recent study was found to be active in vitro, as it induced downregulation of genes critical for the development of MM, including MYC. ${ }^{96}$ The upregulation of $M Y C$, detected either by fluorescent in situ hybridization, gene expression or immunohistochemistry, is seen in up to $50 \%$ of $\mathrm{MM}$ and it has been associated with the development of MM from MGUS, as well as late disease progression. ${ }^{97}$ There are, to date, no known MYC-targeting drugs, and given the importance of MYC upregulation in not only MM but also other tumors, JQ1 could be a future cancer therapy if its efficacy gets confirmed in in vivo models.

Furthermore, miRNA-based therapy is a rapidly evolving field, and development of synthetic oligonucleotides that can either mimic or inhibit miRNAs is widely examined. ${ }^{98}$ Synthetic miRNAs have proven to be effective in $\mathrm{MM}$ in both in vitro and in vivo mouse models. ${ }^{15,99}$ However, systemic administration of miRNAs is a challenging task because of not only the endonuclease cleavage, but also unknown efficacy of delivery to the target cells, issues that can partly be overcome with miRNA nanoparticle conjunction or use of a viral transfection system. ${ }^{98}$ Lastly, there are data supporting that curcumin, a recipient of the Asian spice turmeric, acts as a universal epigenetic modulator, and has antimyeloma activity in vitro and in vivo. ${ }^{100,101}$ There are now ongoing trials evaluating its efficacy in $\mathrm{MM}$, as well as other cancer forms (NCT00113841 and NCT00927485).

Despite the recent progress in understanding the pathogenesis of $\mathrm{MM}$ and the development of novel treatment strategies with longer remission duration, the disease is still considered incurable with the current approaches. Epigenetic aberrations play an important role to the pathogenic events leading to MM, and further studies are needed to elucidate the precise role of, for example, histone modifications and miRNA expression patterns and evaluate their clinical perspective. It is possible that better understanding of the genetic and epigenetic interactions in MM may reveal new understanding of $\mathrm{MM}$ pathogenesis, new disease biomarkers and hopefully the development of novel, individualized treatment strategies, contributing to a more effective disease control.

\section{CONFLICT OF INTEREST}

The authors declare no conflict of interest.

\section{REFERENCES}

1 Palumbo A, Anderson K. Multiple myeloma. N Engl J Med 2011; 364: 1046-1060.

2 Landgren O, Kyle Ra, Pfeiffer RM, Katzmann Ja, Caporaso NE, Hayes RB et al. Monoclonal gammopathy of undetermined significance (MGUS) consistently precedes multiple myeloma: a prospective study. Blood 2009; 113: 5412-5417.

3 Sharma A, Heuck CJ, Fazzari MJ, Mehta J, Singhal S, Greally JM et al. DNA methylation alterations in multiple myeloma as a model for epigenetic changes in cancer. Wiley Interdiscip Rev Syst Biol Med 2010; 2: 654-669.

4 Chapman Ma, Lawrence MS, Keats JJ, Cibulskis K, Sougnez C, Schinzel AC et al. Initial genome sequencing and analysis of multiple myeloma. Nature 2011; 471: 467-472.

5 Dimopoulos K, Gimsing P, Grønbaek K. Aberrant microRNA expression in multiple myeloma. Eur J Haematol 2013; 91: 95-105.

6 Fonseca R, Bergsagel PL, Drach J, Shaughnessy J, Gutierrez N, Stewart aK et al. International Myeloma Working Group molecular classification of multiple myeloma: spotlight review. Leukemia 2009; 23: 2210-2221.

7 Jones Pa. Functions of DNA methylation: islands, start sites, gene bodies and beyond. Nat Rev Genet 2012; 13: 484-492.

8 Walton EL, Francastel C, Velasco G. Maintenance of DNA methylation: Dnmt3b joins the dance. Epigenetics 2011; 6: 1373-1377.

9 Williams K, Christensen J, Helin K. DNA methylation: TET proteins-guardians of CpG islands? EMBO Rep 2012; 13: 28-35.

10 Grønbaek K, Hother C, Jones PA. Epigenetic changes in cancer. APMIS 2007; 115: 1039-1059.

11 Walker Ba, Wardell CP, Chiecchio L, Smith EM, Boyd KD, Neri A et al. Aberrant global methylation patterns affect the molecular pathogenesis and prognosis of multiple myeloma. Blood 2011; 117: 553-562.

12 Heuck CJ, Mehta J, Bhagat T, Gundabolu K, Yu Y, Khan S et al. Myeloma is characterized by stage-specific alterations in DNA methylation that occur early during myelomagenesis. J Immunol 2013; 190: 2966-2975.

13 Kaiser MF, Johnson DC, Wu P, Walker Ba, Brioli A, Mirabella $F$ et al. Global methylation analysis identifies prognostically important epigenetically inactivated tumour suppressor genes in multiple myeloma. Blood 2013; 122 219-226.

14 Bollati V, Fabris S, Pegoraro V, Ronchetti D, Mosca L, Deliliers GL et al. Differential repetitive DNA methylation in multiple myeloma molecular subgroups. Carcinogenesis 2009; 30: 1330-1335.

15 Amodio N, Leotta M, Bellizzi D, Di Martino MT, D'Aquila P, Lionetti $M$ et al. DNA-demethylating and anti-tumor activity of synthetic miR-29b mimics in multiple myeloma. Oncotarget 2012; 3: 1246-1258.

16 Xu W, Yang H, Liu Y, Yang Y, Wang P, Kim S-H et al. Oncometabolite 2-hydroxyglutarate is a competitive inhibitor of $\alpha$-ketoglutarate-dependent dioxygenases. Cancer Cell 2011; 19: 17-30.

17 Song SJ, Ito K, Ala U, Kats L, Webster K, Sun SM et al. The oncogenic microRNA miR-22 targets the TET2 tumor suppressor to promote hematopoietic stem cell self-renewal and transformation. Cell Stem Cell 2013; 13: 87-101.

18 Li Z, Cai X, Cai C-L, Wang J, Zhang W, Petersen BE et al. Deletion of Tet2 in mice leads to dysregulated hematopoietic stem cells and subsequent development of myeloid malignancies. Blood 2011; 118: 4509-4518.

19 Zhou Y, Chen L, Barlogie B, Stephens O, Wu X, Williams DR et al. High-risk myeloma is associated with global elevation of miRNAs and overexpression of EIF2C2/AGO2. Proc Natl Acad Sci USA 2010; 107: 7904-7909.

20 Struhl K, Segal E. Determinants of nucleosome positioning. Nat Struct Mol Biol 2013; 20: 267-273.

21 Kouzarides T. Chromatin modifications and their function. Cell 2007; 128: 693-705.

22 Grunstein M. Histone acetylation in chromatin structure and transcription. Nature 1997; 389: 349-352.

23 Barski A, Cuddapah S, Cui K, Roh T-Y, Schones DE, Wang Z et al. High-resolution profiling of histone methylations in the human genome. Cell 2007; 129: 823-837. 
24 Schwartz YB, Pirrotta V. Polycomb complexes and epigenetic states. Curr Opin Cell Biol 2008; 20: 266-273.

25 Kondo Y, Shen L, Cheng AS, Ahmed S, Boumber Y, Charo C et al. Gene silencing in cancer by histone $\mathrm{H} 3$ lysine 27 trimethylation independent of promoter DNA methylation. Nat Genet 2008; 40: 741-750.

26 Ernst J, Kellis M. Discovery and characterization of chromatin states for systematic annotation of the human genome. Nat Biotechnol 2010; 28: 817-825.

27 Ucar D, Hu Q, Tan K. Combinatorial chromatin modification patterns in the human genome revealed by subspace clustering. Nucleic Acids Res 2011; 39: 4063-4075.

28 Linghu $C$, Zheng $H$, Zhang $L$, Zhang J. Discovering common combinatorial histone modification patterns in the human genome. Gene 2013; 518: 171-178.

29 Cai Y, Geutjes E-J, de Lint K, Roepman P, Bruurs L, Yu L-R et al. The NuRD complex cooperates with DNMTs to maintain silencing of key colorectal tumor suppressor genes. Oncogene 2014; 33: 2157-2168.

30 Plass C, Pfister SM, Lindroth AM, Bogatyrova O, Claus R, Lichter P. Mutations in regulators of the epigenome and their connections to global chromatin patterns in cancer. Nat Rev Genet 2013; 14: 765-780.

31 Croonquist $\mathrm{Pa}$, Van Ness $\mathrm{B}$. The polycomb group protein enhancer of zeste homolog $2(E Z H 2)$ is an oncogene that influences myeloma cell growth and the mutant ras phenotype. Oncogene 2005; 24: 6269-6280.

32 Sander S, Bullinger L, Klapproth K, Fiedler K, Kestler Ha, Barth TFE et al. MYC stimulates EZH2 expression by repression of its negative regulator miR-26a. Blood 2008; 112: 4202-4212.

33 Pichiorri F, Suh S-S, Ladetto M, Kuehl M, Palumbo T, Drandi D et al. MicroRNAs regulate critical genes associated with multiple myeloma pathogenesis. Proc NatI Acad Sci USA 2008; 105: 12885-12890.

34 Stec I, Wright TJ, van Ommen GJ, de Boer Pa, van Haeringen a, Moorman a F et al. WHSC1, a 90kb SET domain-containing gene, expressed in early development and homologous to a Drosophila dysmorphy gene maps in the Wolf-Hirschhorn syndrome critical region and is fused to $\mathrm{lgH}$ in $\mathrm{t}(4 ; 14)$ multiple myeloma. Hum Mol Genet 1998; 7: 1071-1082.

35 Marango J, Shimoyama M, Nishio H, Meyer Ja, Min D-J, Sirulnik A et al. The MMSET protein is a histone methyltransferase with characteristics of a transcriptional corepressor. Blood 2008; 111: 3145-3154.

36 Pei H, Zhang L, Luo K, Qin Y, Chesi M, Fei F et al. MMSET regulates histone H4K20 methylation and 53BP1 accumulation at DNA damage sites. Nature 2011; 470: 124-128.

37 Martinez-Garcia E, Popovic R, Min D-J, Sweet SMM, Thomas PM, Zamdborg $L$ et al. The MMSET histone methyl transferase switches global histone methylation and alters gene expression in $\mathrm{t}(4 ; 14)$ multiple myeloma cells. Blood 2011; 117: 211-220.

38 Kuo AJ, Cheung P, Chen K, Zee BM, Kioi M, Lauring J et al. NSD2 links dimethylation of histone $\mathrm{H} 3$ at lysine 36 to oncogenic programming. Mol Cell 2011; 44: 609-620.

39 Huang Z, Wu H, Chuai S, Xu F, Yan F, Englund N et al. NSD2 is recruited through its PHD domain to oncogenic gene loci to drive multiple myeloma. Cancer Res 2013; 73: 6277-6288.

40 Schmitges FW, Prusty AB, Faty M, Stützer A, Lingaraju GM, Aiwazian J et al. Histone methylation by PRC2 is inhibited by active chromatin marks. Mol Cell 2011; 42: 330-341.

41 Yang P, Guo L, Duan ZJ, Tepper CG, Xue L, Chen X et al. Histone methyltransferase NSD2/MMSET mediates constitutive NF- $\kappa B$ signaling for cancer cell proliferation, survival, and tumor growth via a feed-forward loop. Mol Cell Biol 2012; 32: 3121-3131.

42 Djebali S, Davis Ca, Merkel A, Dobin A, Lassmann T, Mortazavi A et al. Landscape of transcription in human cells. Nature 2012; 489: 101-108.

43 Bartel DP. MicroRNAs: target recognition and regulatory functions. Cell 2009; 136: $215-233$.

44 Fabbri M, Garzon R, Cimmino A, Liu Z, Zanesi N, Callegari E et al. MicroRNA-29 family reverts aberrant methylation in lung cancer by targeting DNA methyltransferases 3A and 3B. Proc Natl Acad Sci USA 2007; 104: 15805-15810.

45 Noonan EJ, Place RF, Pookot D, Basak S, Whitson JM, Hirata H et al. miR-449a targets HDAC-1 and induces growth arrest in prostate cancer. Oncogene 2009; 28: $1714-1724$

46 Wong K-Y, Liang R, So C-C, Jin D-Y, Costello JF, Chim C-S. Epigenetic silencing of MIR203 in multiple myeloma. Br J Haematol 2011; 154: 569-578.

47 Wong KY, Yim RLH, So CC, Jin D-Y, Liang R, Chim CS. Epigenetic inactivation of the MIR34B/C in multiple myeloma. Blood 2011; 118: 5901-5904.

48 Pichiorri F, Suh S-S, Rocci A, De Luca L, Taccioli C, Santhanam R et al. Downregulation of p53-inducible microRNAs 192, 194, and 215 impairs the p53/ MDM2 autoregulatory loop in multiple myeloma development. Cancer Cell 2010; 18: $367-381$.
49 Chi J, Ballabio E, Chen X-H, Kušec R, Taylor S, Hay D et al. MicroRNA expression in multiple myeloma is associated with genetic subtype, isotype and survival. Biol Direct 2011; 6: 23.

50 Corthals SL, Sun SM, Kuiper R, de Knegt Y, Broyl a, van der Holt B et al. MicroRNA signatures characterize multiple myeloma patients. Leukemia 2011; 25: $1784-1789$.

51 Munker R, Liu C-G, Taccioli C, Alder H, Heerema N. MicroRNA profiles of drugresistant myeloma cell lines. Acta Haematol 2010; 123: 201-204.

52 Ronchetti D, Todoerti K, Tuana G, Agnelli L, Mosca L, Lionetti M et al. The expression pattern of small nucleolar and small Cajal body-specific RNAs characterizes distinct molecular subtypes of multiple myeloma. Blood Cancer J 2012; 2: e96.

53 Stott FJ, Bates S, James MC, McConnell BB, Starborg M, Brookes S et al. The alternative product from the human CDKN2A locus, p14(ARF), participates in a regulatory feedback loop with p53 and MDM2. EMBO J 1998; 17: 5001-5014.

54 Deshpande A, Sicinski P, Hinds PW. Cyclins and cdks in development and cancer: a perspective. Oncogene 2005; 24: 2909-2915.

55 Witkiewicz AK, Knudsen KE, Dicker AP, Knudsen ES. The meaning of p16ink4a expression in tumors: Functional significance, clinical associations and future developments. Cell Cycle 2011; 10: 2497-2503.

56 Bergsagel PL, Kuehl WM. Critical roles for immunoglobulin translocations and cyclin D dysregulation in multiple myeloma. Immunol Rev 2003; 194: 96-104.

57 Amodio N, Di Martino MT, Foresta U, Leone E, Lionetti M, Leotta M et al. miR-29b sensitizes multiple myeloma cells to bortezomib-induced apoptosis through the activation of a feedback loop with the transcription factor Sp1. Cell Death Dis 2012; 3: e436.

58 O'Donnell Ka, Wentzel Ea, Zeller Kl, Dang CV, Mendell JT. c-Myc-regulated microRNAs modulate E2F1 expression. Nature 2005; 435: 839-843.

59 Chim CS, Fung TK, Liang R. Disruption of INK4/CDK/Rb cell cycle pathway by gene hypermethylation in multiple myeloma and MGUS. Leukemia 2003; 17: 2533-2535.

60 Mateos MV, García-Sanz R, López-Pérez R, Moro MJ, Ocio E, Hernández J et al. Methylation is an inactivating mechanism of the 16 gene in multiple myeloma associated with high plasma cell proliferation and short survival. $\mathrm{Br} J$ Haematol 2002; 118: 1034-1040.

61 Hatzimichael E, Benetatos L, Dasoula A, Dranitsaris G, Tsiara S, Georgiou I et al. Absence of methylation-dependent transcriptional silencing in TP73 irrespective of the methylation status of the CDKN2A CpG island in plasma cell neoplasia. Leuk Res 2009; 33: 1272-1275.

62 Stanganelli C, Arbelbide J, Fantl DB, Corrado C, Slavutsky I. DNA methylation analysis of tumor suppressor genes in monoclonal gammopathy of undetermined significance. Ann Hematol 2010; 89: 191-199.

63 Seidl S, Ackermann J, Kaufmann H, Keck A, Nösslinger T, Zielinski CC et al. DNA-methylation analysis identifies the E-cadherin gene as a potential marker of disease progression in patients with monoclonal gammopathies. Cancer 2004; 100: 2598-2606.

64 Gonzalez-Paz N, Chng WJ, McClure RF, Blood E, Oken MM, Van Ness B et al. Tumor suppressor p16 methylation in multiple myeloma: biological and clinical implications. Blood 2007; 109: 1228-1232.

65 Niehrs C. The complex world of WNT receptor signalling. Nat Rev Mol Cell Biol 2012; 13: 767-779.

66 Klaus A, Birchmeier W. Wnt signalling and its impact on development and cancer. Nat Rev Cancer 2008; 8: 387-398.

67 Martello G, Zacchigna L, Inui M, Montagner M, Adorno M, Mamidi A et al. MicroRNA control of Nodal signalling. Nature 2007; 449: 183-188.

68 Roccaro AM, Sacco A, Thompson B, Leleu X, Azab AK, Azab F et al. MicroRNAs $15 \mathrm{a}$ and 16 regulate tumor proliferation in multiple myeloma. Blood 2009; 113: 6669-6680.

69 Sun C-Y, She X-M, Qin Y, Chu Z-B, Chen L, Ai L-S et al. miR-15a and miR-16 affect the angiogenesis of multiple myeloma by targeting VEGF. Carcinogenesis 2012; 00: 1-10.

70 Kawano Y, Kypta R. Secreted antagonists of the Wnt signalling pathway. J Cell Sci 2003; 116(Pt 13): 2627-2634.

71 Tian E, Zhan F, Walker R, Rasmussen E, Ma Y, Barlogie B et al. The role of the Wnt-signaling antagonist DKK1 in the development of osteolytic lesions in multiple myeloma. N Engl J Med 2003; 349: 2483-2494.

72 Cha YH, Kim NH, Park C, Lee I, Kim HS, Yook Jl. MiRNA-34 intrinsically links p53 tumor suppressor and Wnt signaling. Cell Cycle 2012; 11: $1273-1281$.

73 Huang K, Zhang J-X, Han L, You Y-P, Jiang T, Pu P-Y et al. MicroRNA roles in beta-catenin pathway. Mol Cancer 2010; 9: 252.

74 Onder TT, Gupta PB, Mani Sa, Yang J, Lander ES, Weinberg RA. Loss of E-cadherin promotes metastasis via multiple downstream transcriptional pathways. Cancer Res 2008; 68: 3645-3654. 
75 Kam Y, Quaranta V. Cadherin-bound beta-catenin feeds into the Wnt pathway upon adherens junctions dissociation: evidence for an intersection between beta-catenin pools. PLoS One 2009; 4: e4580.

76 Chim C-S, Liang R, Leung M-H, Yip S-F, Kwong Y-L. Aberrant gene promoter methylation marking disease progression in multiple myeloma. Leukemia 2006; 20: 1190-1192.

77 Cao M, Seike M, Soeno C, Mizutani $H$, Kitamura $K$, Minegishi $Y$ et al. MiR-23a regulates TGF- $\beta$-induced epithelial-mesenchymal transition by targeting E-cadherin in lung cancer cells. Int $J$ Oncol 2012; 41: 869-875.

78 Heinrich PC, Behrmann I, Haan S, Hermanns HM, Müller-Newen G, Schaper F. Principles of interleukin (IL)-6-type cytokine signalling and its regulation. Biochem J 2003; 374(Pt 1): 1-20.

79 Collins AS, McCoy CE, Lloyd AT, O'Farrelly C, Stevenson NJ. miR-19a: an effective regulator of SOCS3 and enhancer of JAK-STAT signalling. PLoS One 2013; 8 : e69090.

80 Xiong $\mathrm{Q}$, Zhong $\mathrm{Q}$, Zhang J, Yang $\mathrm{M}$, Li C, Zheng $\mathrm{P}$ et al. Identification of novel miR-21 target proteins in multiple myeloma cells by quantitative proteomics. J Proteome Res 2012; 11: 2078-2090.

81 Löffler D, Brocke-Heidrich K, Pfeifer G, Stocsits C, Hackermüller J, Kretzschmar AK et al. Interleukin-6 dependent survival of multiple myeloma cells involves the Stat3-mediated induction of microRNA-21 through a highly conserved enhancer. Blood 2007; 110: 1330-1333.

82 Wang X, Li C, Ju S, Wang Y, Wang H, Zhong R. Myeloma cell adhesion to bone marrow stromal cells confers drug resistance by microRNA-21 up-regulation. Leuk Lymphoma 2011; 52: 1991-1998.

83 Xiong H, Du W, Zhang Y-J, Hong J, Su W-Y, Tang J-T et al. Trichostatin A, a histone deacetylase inhibitor, suppresses JAK2/STAT3 signaling via inducing the promoter-associated histone acetylation of SOCS1 and SOCS3 in human colorectal cancer cells. Mol Carcinog 2012; 51: 174-184.

84 Martoriati A, Doumont G, Alcalay M, Bellefroid E, Pelicci PG, Marine J-C. dapk1, encoding an activator of a p19ARF-p53-mediated apoptotic checkpoint, is transcription target of p53. Oncogene 2005; 24: 1461-1466.

85 Fenaux P, Mufti GJ, Hellstrom-Lindberg E, Santini V, Finelli C, Giagounidis A et al. Efficacy of azacitidine compared with that of conventional care regimens in the treatment of higher-risk myelodysplastic syndromes: a randomised, open-label, phase III study. Lancet Oncol 2009; 10: 223-232.

86 Khong T, Sharkey J, Spencer A. The effect of azacitidine on interleukin-6 signaling and nuclear factor-kappaB activation and its in vitro and in vivo activity against multiple myeloma. Haematologica 2008; 93: 860-869.

87 Spange $\mathrm{S}$, Wagner T, Heinzel T, Krämer $\mathrm{OH}$. Acetylation of non-histone proteins modulates cellular signalling at multiple levels. Int J Biochem Cell Biol 2009; 41 185-198.

88 Todoerti K, Barbui V, Pedrini O, Lionetti M, Fossati G, Mascagni $P$ et al. Pleiotropic anti-myeloma activity of ITF2357: inhibition of interleukin-6 receptor signaling and repression of miR-19a and miR-19b. Haematologica 2010 95: 260-269.

89 Lamottke B, Kaiser M, Mieth M, Heider U, Gao Z, Nikolova Z et al. The novel, orally bioavailable HSP90 inhibitor NVP-HSP990 induces cell cycle arrest and apoptosis in multiple myeloma cells and acts synergistically with melphalan by increased cleavage of caspases. Eur J Haematol 2012; 88: 406-415.

90 Richardson PG, Schlossman RL, Alsina M, Weber DM, Coutre SE, Gasparetto $C$ et al. PANORAMA 2: panobinostat in combination with bortezomib and dexamethasone in patients with relapsed and bortezomib-refractory myeloma. Blood 2013; 122: 2331-2337.

91 Dimopoulos M, Siegel DS, Lonial S, Qi J, Hajek R, Facon T et al. Vorinostat or placebo in combination with bortezomib in patients with multiple myeloma (VANTAGE 088): a multicentre, randomised, double-blind study. Lancet Onco 2013; 14: 1129-1140.

92 Offidani M, Polloni C, Cavallo F, Liberati AM, Ballanti S, Pulini S et al. Phase II study of melphalan, thalidomide and prednisone combined with oral panobinostat in patients with relapsed/refractory multiple myeloma. Leuk Lymphoma 2012; 53: 1722-1727.

93 New M, Olzscha H, La Thangue NB. HDAC inhibitor-based therapies: can we interpret the code? Mol Oncol 2012; 6: 637-656.

94 Santo $L$, Hideshima $T$, Kung $A L$, Tseng J, Tamang D, Yang $M$ et al. Preclinical activity, pharmacodynamic, and pharmacokinetic properties of a selective HDAC6 inhibitor, ACY-1215, in combination with bortezomib in multiple myeloma. Blood 2012; 119: 2579-2589.

95 Spannhoff A, Hauser A-T, Heinke R, Sippl W, Jung M. The emerging therapeutic potential of histone methyltransferase and demethylase inhibitors. ChemMedChem 2009; 4: 1568-1582.

96 Lovén J, Hoke Ha, Lin CY, Lau A, Orlando Da, Vakoc CR et al. Selective inhibition of tumor oncogenes by disruption of super-enhancers. Cell 2013; 153: 320-334.

97 Chng W-J, Huang GF, Chung TH, Ng SB, Gonzalez-Paz N, Troska-Price T et al. Clinical and biological implications of MYC activation: a common difference between MGUS and newly diagnosed multiple myeloma. Leukemia 2011; 25: 1026-1035.

98 lorio MV, Croce CM. MicroRNA dysregulation in cancer: diagnostics, monitoring and therapeutics. A comprehensive review. EMBO Mol Med 2012; 4: 143-159.

99 Di Martino MT, Leone E, Amodio N, Foresta U, Lionetti M, Pitari MR et al. Synthetic miR-34a mimics as a novel therapeutic agent for multiple myeloma: in vitro and in vivo evidence. Clin Cancer Res 2012; 18: 6260-6270.

100 Fu S, Kurzrock R. Development of curcumin as an epigenetic agent. Cancer 2010; 116: 4670-4676.

101 Sung B, Kunnumakkara AB, Sethi G, Anand P, Guha S, Aggarwal BB. Curcumin circumvents chemoresistance in vitro and potentiates the effect of thalidomide and bortezomib against human multiple myeloma in nude mice model. Mol Cancer Ther 2009; 8: 959-970.

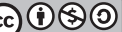

This work is licensed under a Creative Commons AttributionNonCommercial-ShareAlike 3.0 Unported License. The images or other third party material in this article are included in the article's Creative Commons license, unless indicated otherwise in the credit line; if the material is not included under the Creative Commons license, users will need to obtain permission from the license holder to reproduce the material. To view a copy of this license, visit http:// creativecommons.org/licenses/by-nc-sa/3.0/ 